\title{
Fluorescein Angiography Compared to Three-Dimensional Measurements by the Retinal Thickness Analyzer in Classic Choroidal Neovascularization
}

\author{
R.W. Strauss ${ }^{\text {a }} \quad$ F. Rombold ${ }^{\mathrm{a}, \mathrm{b}} \quad$ A. Kampik ${ }^{\mathrm{a}} \quad$ A.S. Neubauer ${ }^{\mathrm{a}}$ \\ a Department of Ophthalmology, Ludwig-Maximilians University, Munich, and ${ }^{\mathrm{b}}$ Department of Ophthalmology, \\ Zentralklinikum Augsburg, Augsburg, Germany
}

\section{Key Words}

Age-related macular degeneration $\cdot$ Choroidal

neovascularization $\cdot$ Macular imaging $\cdot$ Photodynamic therapy

\begin{abstract}
Purpose: To compare and correlate imaging of classic subfoveal choroidal neovascularization (CNV) with noninvasive 3dimensional imaging by the retinal thickness analyzer (RTA) to conventional fluorescein angiography (FA). Methods: A total of 29 eyes of 29 consecutive patients with predominantly classic CNV eligible for photodynamic therapy underwent FA and RTA imaging. The FA dimensions of the CNV were measured independently by two graders. With the RTA, masked to FA the size of the CNV itself as imaged in 3dimensional reconstruction, the size of significantly thickened retina overlying the CNV and the maximum retinal thickness were measured. Results: The mean diameter of the CNV determined from 3-dimensional RTA reconstructions showed an excellent correlation with measurements from FA $(r=0.91, p<0.001)$. The area of retinal thickening was by a mean of $0.7 \mathrm{~mm}$ in diameter larger and correlated moderately well with the size of the CNV on FA $(r=0.65, p<$ 0.001 ). In contrast, there was no correlation between the absolute retinal thickness and the CNV size on FA. Conclusions:
\end{abstract}

Noninvasive quantitative mapping of predominantly classic CNV by RTA is feasible and also allows 3-dimensional measurement of the lesion itself. The results correlate well with FA assessment but visualize different properties of the disease.

Copyright $\odot 2007$ S. Karger AG, Basel

\section{Introduction}

Age-related macular degeneration is a leading cause of legal blindness in the Western World with an increasing incidence and prevalence $[1,2]$. Especially the 'wet forms' due to choroidal neovascularization (CNV) account for the majority of rapid and severe visual loss. Based on the fluorescein angiography (FA) appearance, CNVs are currently subdivided into occult and classic forms. From a well-established therapy for classic membranes, photodynamic therapy (PDT), this subtype is known to exhibit typical biological features making it treatable by PDT. Due to relatively high recurrence rates, a close follow-up is needed, conventionally performed by FA. Less invasive and faster diagnostic approaches for diagnosis and follow-up are desirable, which might be obtained by crosssectioning imaging devices such as optical coherence tomography (OCT, Carl-Zeiss Meditec, Jena, Germany) or

\section{KARGER \\ Fax +4161306 1234 \\ E-Mail karger@karger.ch}

www.karger.com
(C) 2007 S. Karger AG, Basel

0030-3747/07/0392-0098\$23.50/0

Accessible online at:

www.karger.com/ore
Aljoscha S. Neubauer, MD

Department of Ophthalmology, Ludwig-Maximilians University Munich

DE-80336 München (Germany)

Tel. +49 895160 3811, Fax +498951605160

E-Mail aljoscha.neubauer@med.uni-muenchen.de 
the retinal thickness analyzer (RTA). The RTA (Talia Technology, Lod Indst. Area, Israel) is an imaging device originally designed for the mapping of macular edema and objective measurements of retinal thickness at the posterior pole [3-6]. The RTA calculates the retinal thickness from the difference between the vitreoretinal and the chorioretinal surface. Imaging is fast enough to avoid any motion artifacts and covers an area of $3 \times 3 \mathrm{~mm}$ with each scan. Consecutively, also 3-dimensional mapping of the retina and retinal pigment epithelium (RPE) layer can be performed by the same technology [7] including Cscan-like viewing and slicing of the resulting voxel block. Imaging classic $\mathrm{CNV}$ with the instrument might facilitate follow-up and might reduce the number of FA examinations necessary, if sufficient information could be obtained. Therefore as a fist step the current study investigates the correlation of cross-sectional and 3-dimensional RTA imaging of classic CNVs with their fluorescein angiographic properties.

\section{Methods}

\section{Patients}

Twenty-nine eyes of 29 consecutive patients with predominantly classic, subfoveal CNV due to age-related macular degeneration eligible for PDT according to the original TAP criteria [8] were included. Patients were also eligible when PDT was performed for classic but juxtafoveal CNV [9]. All patients underwent conventional FA and RTA scanning on the same day in random order at the Department of Ophthalmology, LudwigMaximilians-Universität, Munich, Germany. Eyes with other coexisting retinal disease or any significant media opacities were excluded. Research was conducted after informed consent had been obtained from each patient and conformed to the tenets of the World Medical Association Declaration of Helsinki. In total, there were 12 right and 17 left eyes of 29 patients assigned to this study (11 male, 18 female). In the highly selected group of patients eligible for PDT by the criteria given above, no eyes had to be excluded, especially not for image quality of any instrument. Age ranged from 60 to 84 years with a mean age of $75 \pm 9.1$ years $( \pm$ standard deviation). Refraction ranged in spherical equivalent between -5 and $+4.5 \mathrm{dpt}$. Best corrected visual acuity ranged from $20 / 200$ to $20 / 40$ (median 20/100).

\section{Fluorescein Angiography}

Routine FA was performed after rapid injection of $5 \mathrm{ml}$ of a $20 \%$ solution of fluorescein (Ciba Vision Ophthalmics, Toulouse, France). A series of digital fundus pictures was taken with a scanning laser ophthalmoscope (Heidelberg Retina Angiograph; Heidelberg Engineering, Heidelberg, Germany). From each series, a picture from the early phase $(10-20 \mathrm{~s})$ and a picture from the later phase (3-5 min) showing the sharpest boundaries of the lesion were selected for analysis (fig. 1A, B). The CNV was defined to be classic if a discrete, well-demarcated focal area of hyperfluores- cence was detectable in the early phase with fluorescein leakage in late frames [11]. Analysis of fluorescein angiograms consisted of measurement of the CNV dimensions (maximum diameter with all directions considered) independently by two masked graders. The mean of the two graders was used as a reference, absolute sizes were calculated by the Heidelberg Retina Angiograph's proprietary software program taking into account an appropriate magnification factor dependent on the instrument and characteristics of the eye such as refraction [12].

\section{RTA Measurements}

All patients were scanned with the RTA equipped with a color fundus camera. The principles of measurement have been described in detail elsewhere $[5,6]$. With the instrument version used, 16 optical cross-section images covering an area of $3 \times$ $3 \mathrm{~mm}$ on the retina are acquired in $300 \mathrm{~ms}$ during 1 scan. These optical sections are thus spaced approximately $190 \mu \mathrm{m}$ apart. The posterior pole is completely covered in 5 overlapping scans and a retinal thickness map is automatically composed (fig. 1C). For the current study the instrument's equidistant quadratic coverage of the posterior pole with several motion-free scans is important, which allows 3-dimensional display and analysis of the resulting voxel blocks. Appropriate fixation was verified by observing a fundus image (and the projection and recording of the fixation mark) that is acquired simultaneously. Analysis consisted of absolute maximum retinal thickness measurements (intraretinal edema overlying the CNV), the area of abnormal thickening and finally assessment of the size of the CNV. Of these the maximum retinal thickness and the area of significant thickening were calculated by the instrument's built-in software (version 4.11B). Maximum thickness and the maximal diameter of significant retinal thickening (outside the instrument's normative database) were obtained from the standard thickness maps.

The size of the CNV (maximum diameter) was assessed from the 3-dimensional display of the slit images of the RTA in a masked manner by one experienced observer (R.W.S.). This was performed in detail by determining the extension of higher reflectivity and anterior displacement at the posterior part of the slits, which corresponds to the RPE level. Such higher reflectivity at the posterior RPE level is assumed to be caused by the CNV itself $[10,13]$ and does not include exudation. Therefore, those measurements should correspond to the real maximum CNV diameter. Manual measurements were performed on 3-dimensional and reversely displayed data sets with the instrument's Anatomy Imager software. An example is shown in figure 1 illustrating the highly reflective $\mathrm{CNV}$ on the cross-section slits (fig. 1D) and their viewing in 3-dimensional display (fig. 1E), which greatly facilitates defining the borders to measure $\mathrm{CNV}$ size. If the identified lesion extended over one single $3 \times 3 \mathrm{~mm}$ RTA scan, which occurred in 7/29 eyes, the maximum extension on both adjacent scans was measured and added. This is possible, as the scans are registered by the instrument's software on the fundus image allowing exact localization. In no case did the lesion extend over the $6 \times 6 \mathrm{~mm}$ area of the posterior pole covered by RTA scanning. While the resolution of thickness measurements in the $x$-y plane over the posterior pole is $190 \mu \mathrm{m}$, the 3-dimensional display technique as applied here may have a much higher spatial resolution - at least in the y-axis direction where the complete slit images are shown with 256 pixels over 3 $\mathrm{mm}$ distance, i.e. $11.7 \mu \mathrm{m}$ spaced apart. As the simultaneously 
Fig. 1. Example of a predominantly classic CNV. A Early phase of the fluorescein angiogram. Note the well-demarcated area of hyperfluorescence indicating the area of neovascularization which measured a maximum diameter of $1.46 \mathrm{~mm}$. B Late phase of FA with increasing leakage at the site of the lesion. C Retinal thickness deviation from the normal database of the RTA. The diameter of significant thickening was determined on this map, in this case $1.5 \mathrm{~mm}$ horizontally as measured in the instrument's analysis software. Horizontal and vertical measurements were performed on the deviation map for comparison with FA. D Slit image of the RTA center scan, encompassing an area of $3 \times$ $3 \mathrm{~mm}$. The CNV is encircled in yellow by determining the maximum diameter of higher reflectivity at the posterior part of the slit (to the right, corresponding to the RPE level), which is assumed to be caused by or to represent the CNV [10]. The diameter of altered reflectivity measured manually in the slit analysis of the RTA software yields a maximum of $1.5 \mathrm{~mm}$. Although this method is theoretically feasible, measurements are much facilitated by the 3-dimensional display technique as shown in E. E Three-dimensional display of the same $3 \times 3 \mathrm{~mm}$ area as in $\mathbf{D}$. The 3-dimensional voxel block is viewed from the chorioretinal side ('upside down') with the Anatomy Imager software. The chorioretinal side is slightly sectioned while viewed from downside to define the extent of the classic CNV in the left part of the image. It measures approximately 1.5 $\mathrm{mm}$ in maximum diameter, a caliper is shown.
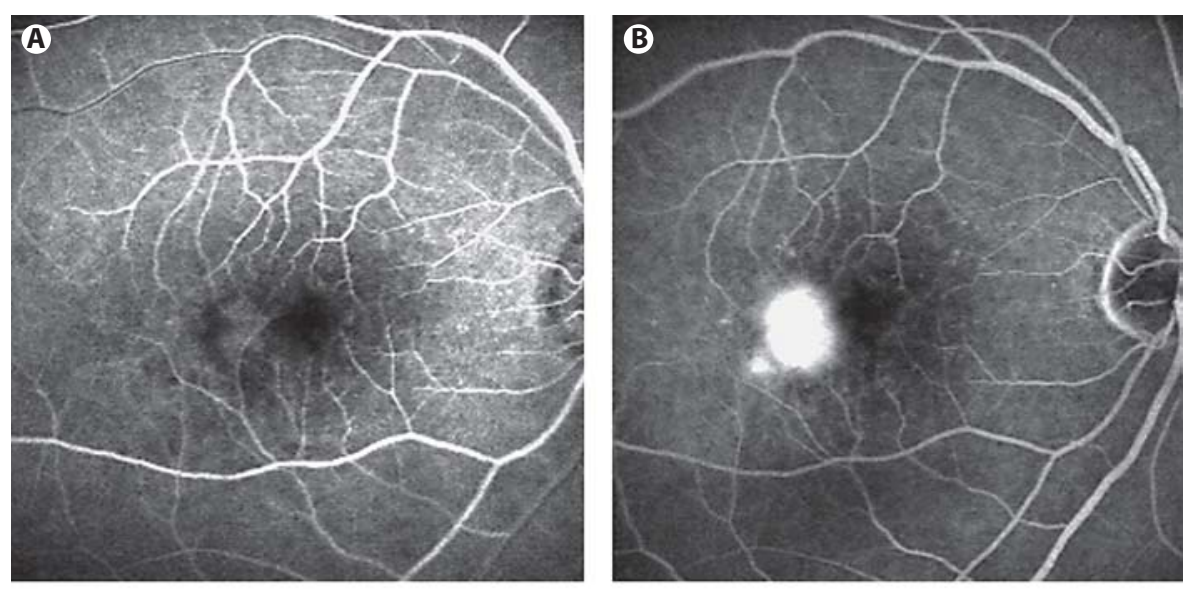

C Higher
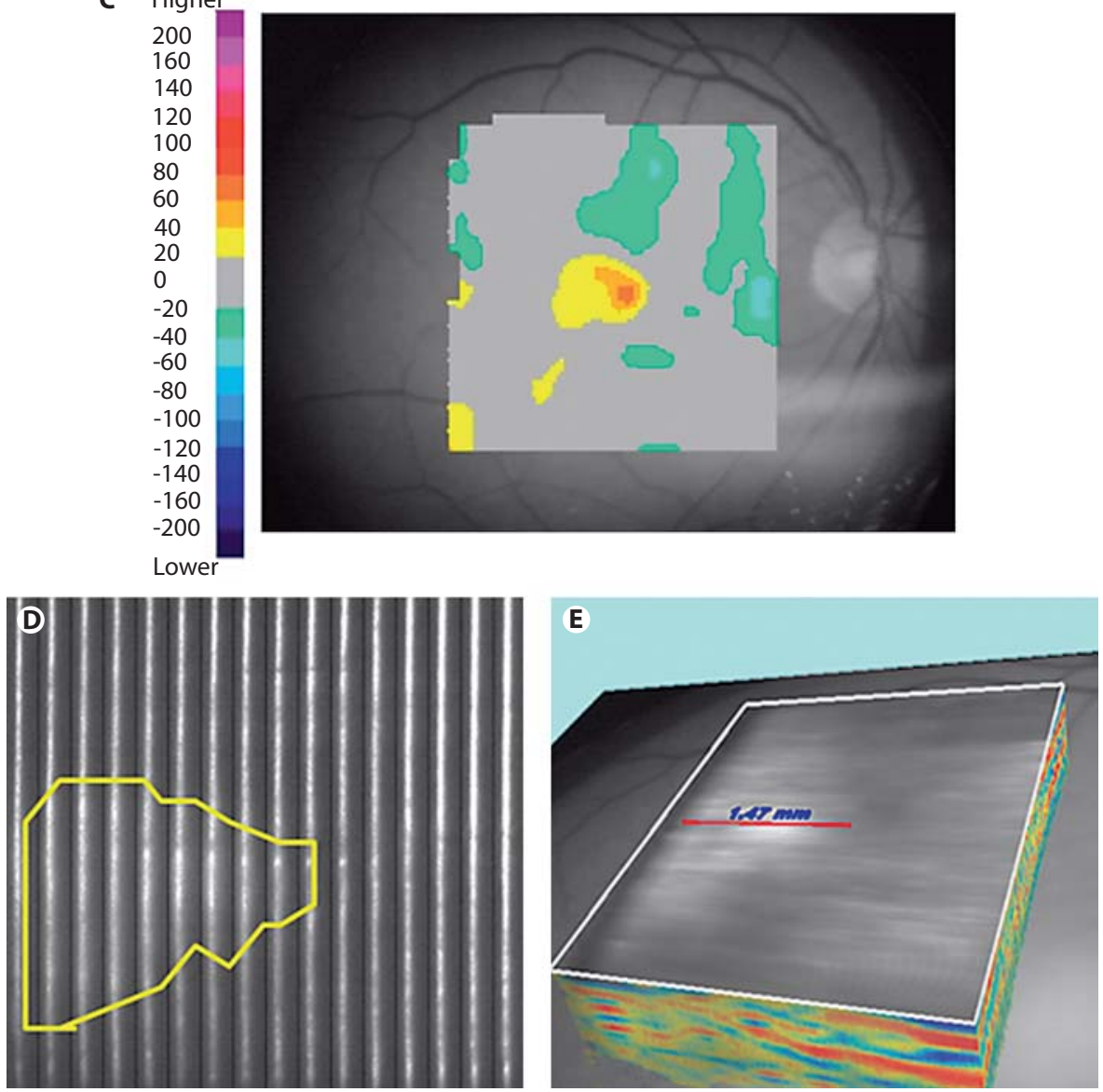

taken color fundus photograph can be superimposed on the 3dimensional voxel block, the $\mathrm{x}-\mathrm{y}$ resolution might be improved by this means for clinical measurements related to the retinal surface - a technique not used in the current study where reverse viewing of the CNV from below was applied. For statistical analysis, SPSS for Windows (SPSS Inc., Chicago, Ill., USA), version 12.0, was used; $\mathrm{p}<0.05$ was considered significant and $\mathrm{p} \geq 0.05$ nonsignificant (n.s.).

\section{Results}

The maximum diameter of the CNV determined by FA was obtained from two graders, which showed an excellent correlation $(\mathrm{r}=0.918, \mathrm{p}<0.001)$; its mean was 2.3 $\pm 0.9 \mathrm{~mm}$ ( \pm standard deviation). The maximum diameter of the CNV as determined from RTA imaging was 


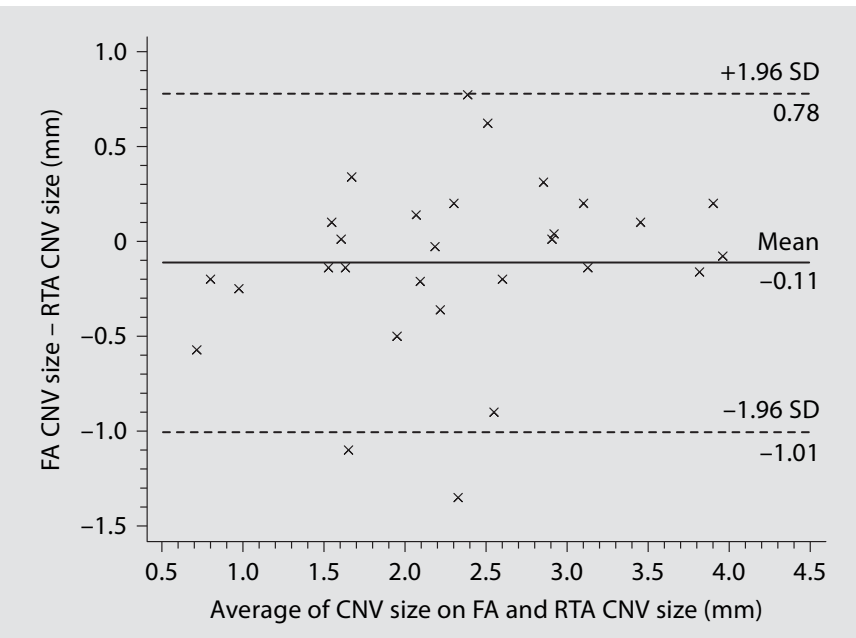

Fig. 2. Bland-Altman plot of the maximum CNV diameter as determined from the RTA 3-dimensional image analysis versus maximum diameter of the $\mathrm{CNV}$ on FA. An excellent correlation of the size determined from the RTA with the CNV size on FA was observed $(r=0.91, p<0.001)$, means do not differ significantly $(p=0.19)$. However, in some cases distinct differences of up to $1.35 \mathrm{~mm}$ exist. $\mathrm{SD}=$ Standard deviation.

not statistically different with a mean of $2.5 \pm 1.0 \mathrm{~mm}$ $(\mathrm{p}=0.19)$. The $\mathrm{CNV}$ diameters obtained from the two imaging modalities correlated well with each other $(\mathrm{r}=$ $0.91, \mathrm{p}<0.001$ ) as shown in figure 2. Detailed analysis of their differences yielded a significant but moderate correlation $(\mathrm{r}=0.46, \mathrm{p}=0.01)$ of the method difference with the size of the lesion as defined on FA: in small lesions the RTA CNV measurements tended to be relatively too large, while larger lesions showed fewer differences between the two modalities (n.s. for the larger half of the lesions). The difference in size between the two modalities also correlated significantly with the classic percentage of the $\mathrm{CNV}$ $(\mathrm{r}=-0.40, \mathrm{p}=0.03)$ : more occult lesions showed higher differences between the two imaging modalities than more classic lesions.

As shown in figure 3, the area of significant retinal thickening showed a significantly $(\mathrm{p}<0.001)$ larger diameter on RTA $(3.0 \pm 1.2 \mathrm{~mm})$ than on FA $(2.3 \pm 0.9$ $\mathrm{mm})$, but on the other hand the diameter on FA and the thickened retina area correlated well $(\mathrm{r}=0.61, \mathrm{p}<0.001)$. The existing absolute differences between the diameter of the thickened retina on RTA and on FA correlated with the size of the thickened retina area as measured by RTA $(\mathrm{r}=0.65, \mathrm{p}<0.001)$ : for larger areas of thickening the RTA showed higher values and larger differences compared to the FA diameter (fig. 3). However, an inverse, nonsignif-



Fig. 3. Maximum CNV diameter on FA versus the maximum diameter of thickened retina as measured with the RTA. The thickened retina measured on RTA was by a mean of $0.7 \mathrm{~mm}$ (dotted line) significantly larger $(\mathrm{p}=0.001)$ than the CNV on FA, but a moderately good correlation with FA exists $(r=0.65, p<$ 0.001).

icant correlation was observed when the CNV size was defined by FA. In summary no definitive factor responsible for the degree of discordance between the two imaging modalities could be identified. Regarding retinal thickness (z-direction), the maximum absolute retinal thickness overlying the CNV measured a mean of $373 \pm$ $93 \mu \mathrm{m}$ and did not correlate with the size of the CNV on FA $(r=0.1, p=0.568)$.

\section{Discussion}

We could demonstrate that measuring classic CNV in age-related macular degeneration is feasible with a crosssectioning noninvasive imaging device, the RTA, and that CNV size measurements (at the RPE level) correlate well with those of FA. However, the area of retinal thickening tends to be larger than that of the $\mathrm{CNV}$, which may be explained by retinal edema adjacent to the CNV. Fluid accumulation, debris deposits, location of the CNV relative to the RPE and sensory retina, scarring, tissue atrophy (especially after PDT) and other factors may cause the area of retinal thickening to be larger than the CNV seen on FA. Peak retinal thickness does not correlate with the CNV size or any other parameter investigated: it may therefore rather reflect different amounts of leakage and 
might to some degree correspond with the activity of the lesion. Those results are in concordance with those from other groups investigating FA compared to OCT $[13,14]$. OCT and the RTA as applied in our study are known to give similar results in CNV imaging [15].

For the first time our study systematically quantifies the size of the CNV on cross-section and 3-dimensional reconstructed (RTA) images, for which an excellent correlation with FA could be shown. The 3-dimensional display of the RTA data set in a C-scan-like fashion with the Anatomy Imager much facilitates correctly defining the area of the CNV. This is in agreement with studies applying OCT cross-sectioning, where OCT was found to be even more reliable than FA and indocyanine green angiography [10] and more recently OCT 3-dimensional imaging [16]. One might speculate that the differences found between FA and morphological imaging - such as OCT or RTA - may rather reflect the different biological properties of the CNV than errors of either morphological or FA imaging [14, 17]. Approaches to use the knowledge of those differences to modify e.g. PDT treatment regimens are promising [18].

\section{Conclusions}

In summary, noninvasive quantitative mapping of predominantly classic CNV by RTA is feasible and by 3dimensional viewing also allows measurement of the size of the lesion itself. The results correlate well with FA assessment but visualize different properties of the disease.

\section{References}

1 Hawkins BS, Bird A, Klein R, West SK: Epidemiology of age-related macular degeneration. Mol Vis 1999;5:26.

2 Vingerling JR, Dielemans I, Hofman A, Grobbee DE, Hijmering M, Kramer CF, de Jong PT: The prevalence of age-related maculopathy in the Rotterdam Study. Ophthalmology 1995; 102:205-210.

3 Oshima Y, Emi K, Yamanishi S, Motokura M: Quantitative assessment of macular thickness in normal subjects and patients with diabetic retinopathy by scanning retinal thickness analyser. Br J Ophthalmol 1999;83:54-61.

4 Shahidi M, Ogura Y, Blair NP, Rusin MM, Zeimer R: Retinal thickness analysis for quantitative assessment of diabetic macular edema. Arch Ophthalmol 1991;109:11151119.

5 Zeimer R, Shahidi M, Mori M, Zou S, Asrani S: A new method for rapid mapping of the retinal thickness at the posterior pole. Invest Ophthalmol Vis Sci 1996;37:1994-2001.

6 Zeimer RC, Mori MT, Khoobehi B: Feasibility test of a new method to measure retinal thickness noninvasively. Invest Ophthalmol Vis Sci 1989;30:2099-2105.

7 Neubauer AS, Thiel M, Priglinger S, Chryssafis C, Alge C, Welge-Lussen U, Kampik A: Mapping of the retinal pigment epithelium in exudative age related macular degeneration. Graefes Arch Clin Exp Ophthalmol 2006;244:342-351.
8 TAP Study Group: Photodynamic therapy of subfoveal choroidal neovascularization in age-related macular degeneration with verteporfin: one-year results of 2 randomized clinical trials - TAP report 1. Arch Ophthalmol 1999;117:1329-1345.

9 Blair MP, Apte RS, Miskala PH, Bressler SB, Goldberg MF, Schachat AP, Bressler NM: Retrospective case series of juxtafoveal choroidal neovascularization treated with photodynamic therapy with verteporfin. Retina 2004;24:501-506.

10 Kim SG, Lee SC, Seong YS, Kim SW, Kwon OW: Choroidal neovascularization characteristics and its size in optical coherence tomography. Yonsei Med J 2003;44:821-827.

11 Bermig J, Tylla H, Jochmann C, Nestler A, Wolf S: Angiographic findings in patients with exudative age-related macular degeneration. Graefes Arch Clin Exp Ophthalmol 2002;240:169-175.

12 Garway-Heath DF, Rudnicka AR, Lowe T, Foster PJ, Fitzke FW, Hitchings RA: Measurement of optic disc size: equivalence of methods to correct for ocular magnification. Br J Ophthalmol 1998;82:643-649.

13 Hughes EH, Khan J, Patel N, Kashani S, Chong NV: In vivo demonstration of the anatomic differences between classic and occult choroidal neovascularization using optical coherence tomography. Am J Ophthalmol 2005;139:344-346.
14 Sahni J, Stanga P, Wong D, Harding S: Optical coherence tomography in photodynamic therapy for subfoveal choroidal neovascularisation secondary to age related macular degeneration: a cross sectional study. $\mathrm{Br} \mathrm{J} \mathrm{Oph-}$ thalmol 2005;89:316-320.

15 Montero JA, Ruiz-Moreno JM: Macular thickness in patients with choroidal neovascularization determined by RTA and OCT3: comparative results. Eye 2005;19:72-76.

16 Pieroni CG, Witkin AJ, Ko TH, Fujimoto JG, Chan A, Schuman JS, Ishikawa H, Reichel E, Duker JS: Ultrahigh resolution optical coherence tomography in non-exudative age related macular degeneration. Br J Ophthalmol 2006;90:191-197.

17 Eter N, Spaide RF: Comparison of fluorescein angiography and optical coherence tomography for patients with choroidal neovascularization after photodynamic therapy. Retina 2005;25:691-696.

18 Krebs I, Binder S, Stolba U, Schmid K, Glittenberg C, Brannath W, Goll A: Optical coherence tomography guided retreatment of photodynamic therapy. $\mathrm{Br} \mathrm{J}$ Ophthalmol 2005;89:1184-1187. 